\title{
Images by Nuclear Magnetic Resonance. A Modified Version of the EPI Method
}

\author{
Simone Souza Ramalho, Nilson Mendes Borges, and Waldemar Wolney Filho \\ Instituto de Física \\ Universidade Federal de Goiás \\ Caixa Postal 131, 74001-970, Goiânia, GO, Brazil
}

Received on 29 August, 2000

\begin{abstract}
It is well known that data accquisition times and image reconstruction procedures in conventional magnetic resonance imaging (MRI) are usually long. For time dependent phenomena, such as sample motion, respiration, blood flow pulsation and others, faster accquision times are desirable to avoid ghosting artifacts. Over the last two decades, a great number of fast and ultra-fast MRI methods have been proposed that greatly reduce the accquision times. One of them is the echo-planar imaging (EPI) method which uses a single excitation scheme to scan entirely the k-space in a very short time. In the initial sections of this work we will supply the reader with a brief description of the image reconstruction procedures for the conventional MRI sequence and four different schemes of the EPI method. However, our main objective is to propose a modified version of the spin-echo EPI method, suggesting a sequence with the use of a constant phase encoding gradient.
\end{abstract}

\section{Introduction}

The principles of the nuclear magnetic resonance imaging (MRI) technique were firstly introduced by Mansfield, Grannell and simultaneously by Lauterbur in the years of 1973,1974 and $1975^{[1-4]}$. Since its invention, the evolution of the technique was very fast, having an immediate application in the medical area as a very powerful tool for visualization of tissue anatomy and structure. Also it has been used as a valuable diagnostic method for investigating malignant tissues, since spinlattice relaxation time in cancerous growths is known to be different from that in normal tissue ${ }^{[5]}$. The MRI method has further found applications in physics, chemistry, material science, biology and biophysics ${ }^{[6]}$. However, the process of obtaining conventional magnetic resonance imaging (CMRI) is slow, when compared with computarized tomography $(\mathrm{CT})$, for instance ${ }^{[7]}$. The times for data acquisition and reconstruction images are long, typically of the order of 10 minutes or more ${ }^{[8]}$. This happens due to the need of long waiting periods for the repetition of the radio-frequency (RF) pulse sequences, which are of the order of the spin-lattice relaxation times $T_{1}$. This effectively reduces its applicability in medical clinics, where time dependent phenomena are normally present, as for exemple in physiologic processes and involuntary patient movement ${ }^{[9]}$. Besides the delay in the acquisition times, in conventional MRI, the physiologic processes as the patient's breathing, heartbeats, peristalsis movement, cardiac arrythimia and sanguine flow, can cause artifacts and blurring that greatly affect the image quality. Many other time dependent events, such as the movement of the walls of the heart or the dynamics of blood flow can only be investigated with the use of the NMR technique if the time of acquisition data is small compared to the duration of such events.

In the last two decades a great number of very fast pulse sequences has been proposed with the objective of extending the applications of MRI. These sequences differ from the conventional ones because they reduce the time of acquisition to values inferior to $100 \mathrm{~ms}$, as the result of the use of a single excitation RF pulse or sequences of multiple pulses of low angle to rotate the magnetization to the plane of the selected slice ${ }^{[8]}$. More recently, using trains of low flip angle RF excitation pulses, it becomes possible to obtain images in times of the order of $10 \mathrm{~ms}$ (ultra-fast images) ${ }^{[10]}$. One of the processes for obtaining fast images by NMR is provided by the so-called EPI (Echo Planar Imaging) method.

This method and four of its variants will be briefly

\footnotetext{
*Present address: Universidade Católica de Goiás
} 
discussed in section 3. A modified version of the method will be presented in section 4 . However, for a better understanding of how the fast and ultra-fast imaging processes work, firstly we will present, in the next section, a short description of the process of data acquisition in the conventional MRI method.

\section{MRI Reconstruction in Two Dimensions}

In a three-dimensional sample, under equilibrium condition, the magnetization is aligned with the direction of the static magnetic field $\mathbf{H}_{0}$, usually applied along the $z$-direction. If the magnetization is disturbed by a selective excitation, the reconstruction of the image can be performed in two dimensions, in the plane of the selected slice. The resulting image is an average of contributions from all the nuclear spins inside a layer of thickness $b$. The image reconstruction requires that the $\mathbf{k}$-space is sampled and a Fourier transformation is performed. The sampling requires the application of appropriated gradients, oriented at will, in the plane of the slice. Such flexibility is one of the great advantages of the MRI method over the computarized tomography by X-ray, for instance, where such choice of freedom is not allowed ${ }^{[11]}$.

In what follows, we will turn our attention to the image reconstrution in the slice plane. In CMRI method, the sampling of the $\mathbf{k}$-space is performed using a finite number of sample points. The way these points are distributed is known as raster ${ }^{[9,12]}$. The raster may be described using polar or cartersian coordinates. In terms of two-dimensional Fourier transform, the simplest way to understand the problem is to use the cartesian raster, due to Kumar, Welti and Ernest ${ }^{[13,14]}$.

When the FID (Free Induction Decay) signal is sampled in the presence of a gradient, the data points are obtained along a single line in the $\mathbf{k}$-space. This line is oriented along one of the cartesian axes and the associated gradient is known as the read gradient. The $x$-coordinate is usually ascribed to this direction. The intercept of this line along an orthogonal axis can be varied by applying another field gradient orthogonal to the first one, by a fixed period of time, before the sampling begins. Since this gradient imparts a phase modulation to the signal, it is named phase encoding gradient. The direction ascribed to this gradient is the $y$-coordinate. The MRI technique is based upon encoding the resonance frequency $\omega$ of the nuclear spins according to their spatial coordinates. Considering a fixed linear gradient $\mathbf{G}$, applied to a sample, the local resonance frequency of the nuclear spins can be defined as a function of the coordinate $\mathbf{r}$ as ${ }^{[8,9]}$

$$
\omega(\mathbf{r})=\gamma H_{0}+\gamma \mathbf{G} \cdot \mathbf{r},
$$

where $\gamma$ is the gyromagnetic ratio of the resonant nu- cleus and $H_{0}$ is the intensity of the static magnetic field. This simple linear relation between the precession frequency $\omega$ and the nuclear spin coordinates, $\mathbf{r}$, is of fundamental importance for the understanding of the imaging principle. If we consider the nuclear spins in an element of volume $d^{3} r$ at position $\mathbf{r}$, with a local spin density $\rho(\mathbf{r})$, the number os spins in this element of volume is $\rho(\mathbf{r}) d^{3} r$. The NMR signal coming from this volume element may be given by

$$
d S(\mathbf{G}, t)=A e^{i\left(\gamma H_{0}+\gamma \mathbf{G} \cdot \mathbf{r}\right) t} e^{-\frac{t}{T_{2}}} \rho(\mathbf{r}) d^{3} r
$$

where $A$ is a constant of proporcionality. The exponential term $e^{-\frac{t}{T_{2}}}$ is the $T_{2}$ decay term that appears as a limiting factor related to the transverse magnetization dephasing. This term can be conveniently neglected, provided that the dephasing of the transverse magnetization, due to the spread in the off resonance frequency $\gamma$ G.r, is much faster than the decay due to the relaxation time $T_{2}$.

When phase-sensitive detection is used to observe the nuclear signal at the receiver coil, the radiofrequency signal is mixed with a reference oscillation and the result is a signal at the difference frequency, a process known as heterodyne mixing. Taking the reference frequency equal to $\omega_{0}=\gamma H_{0}$, the so-called onresonance condition, the signal obtained oscillates at frequency $\gamma$ G.r. This will be in the audio-frequency range and can be addressed to an analogue-to-digital converter (ADC). Relative to the reference frequency $\omega_{0}$, the amplitude signal may be written as

$$
S(t)=A \iiint \rho(\mathbf{r}) e^{i(\gamma \mathbf{G} \cdot \mathbf{r}) t} d^{3} r,
$$

where we neglected the relaxation term due to $T_{2}$. The eqn. (3) shows that the relation between the observed signal $S(t)$ and the nuclear spin density function $\rho(\mathbf{r})$ has the form of a Fourier transformation. To make things more clear, Mansfield ${ }^{[4,15,16]}$ introduced the concept of reciprocal vetor space, $\mathbf{k}$, defined by

$$
\mathbf{k}=\frac{1}{2 \pi} \gamma \mathbf{G} t
$$

This equation shows that the $\mathbf{k}$-space may be transversed by moving either in time or in gradient magnitude. However, the direction of gradient determines the direction of the transverse. Using the definition expressed by eqn. (3) and the concept of Fourier transform and its inverse, we write

$$
\begin{gathered}
S(\mathbf{k})=A \iiint \rho(\mathbf{r}) e^{i(2 \pi \mathbf{k} \cdot \mathbf{r}) t} d^{3} r \\
\rho(\mathbf{r})=A \iiint S(\mathbf{k}) e^{-i(2 \pi \mathbf{k} \cdot \mathbf{r}) t} d^{3} k .
\end{gathered}
$$

These equations show that $S(\mathbf{k})$ and $\rho(\mathbf{r})$ are mutually conjugate and an image of the nuclear spins density 
function can be obtained through a Fourier transform of the NMR signal, acquired in the $\mathbf{k}$-space domain.

In practice, the sampling of the $\mathbf{k}$-space takes place when the signal is sampled at successive time intervals. In other words, $S(\mathbf{k})$ is measured in the time domain while its Fourier transform yields $\rho(\mathbf{r})$ in the frequency domain. In this sense, it is valid to say that there is a correspondence between the real space and frequency, and between the reciprocal space and time ${ }^{[9]}$. Actually, eqn. (4) shows clearly that $\mathbf{G}$ and $t$ are involved in the determination of the $\mathbf{k}$-space.

The real samples are three-dimensional objects. However, only the nuclear spins contained in one slice that is selectively excited, will contribute to the NMR signal. The reconstruction of the image is then performed in the two dimensions of the slice plane by means of a two-dimensional Fourier transform. For exemple, the FID or the echo signal obtained for a selected slice of thickness $b$, cut perpendicular to the $z$-direction, at $z=z_{0}$, is given by ${ }^{[7,8]}$

$$
S\left(k_{x}, k_{y}\right)=A \int_{z_{0}-\frac{b}{2}}^{z_{0}+\frac{b}{2}}\left[\iint \rho(x, y, z) e^{i 2 \pi\left[x k_{x}(t)+y k_{y}(t)\right]} d x d y\right] d z
$$

In eqn. (7), the outer integral, involving the $z$ component, just represents an average across the slice, and can be conveniently ignored. With this, the reconstruction of the averaged planar density function $\rho\left(x, y, z_{0}\right)$ of the nuclear spins into an image is performed by taking the two-dimensional inverse Fourier transform, eqn. (6), now written as

$$
\rho\left(x, y, z_{0}\right)=\iint S\left(k_{x}, k_{y}\right) e^{-i 2 \pi\left[x k_{x}(t)+y k_{y}(t)\right]} d k_{x} d k_{y}=S(t)
$$

where $k_{x}(t)$ and $k_{y}(t)$ are given by:

$$
\begin{aligned}
& k_{x}(t)=\frac{1}{2 \pi} \gamma G_{x} t \\
& k_{y}(t)=\frac{1}{2 \pi} \gamma G_{y} t
\end{aligned}
$$

Since the magnetization is proportional to the spins density distribution function, eqn. (8) is also proportional to the distribution of the magnetization in the selected slice at the time of measurement. The exponential term in this equation is periodic, with period $2 \pi$. This means that the $k$ value describes a wavelength $\lambda=2 \pi / k$. This is the wavelength of the spatial harmonics in which the real object is decomposed. The possible values of $k_{x}$ and $k_{y}$ are found, respectively, in the intervals

$$
\begin{aligned}
& -k_{x, \min } \leq k_{x} \leq k_{x, \max }, \\
& -k_{y, \min } \leq k_{y} \leq k_{y, \max } .
\end{aligned}
$$

According to eqns. (9) and (10), these two equations may be written as

$$
\begin{aligned}
& -\left(\frac{1}{2 \pi}\right) \gamma G_{x} T \frac{\left(N_{r}\right)}{2} \leq k_{x} \leq\left(\frac{1}{2 \pi}\right) \gamma G_{x} T \frac{\left(N_{r}-1\right)}{2} \\
& -\left(\frac{1}{2 \pi}\right) \gamma G_{y} T \frac{\left(N_{p}\right)}{2} \leq k_{y} \leq\left(\frac{1}{2 \pi}\right) \gamma G_{y} T \frac{\left(N_{p}-1\right)}{2}
\end{aligned}
$$

where $N_{r}$ is the number of points per profile, $N_{p}$ is the number of phase encoding gradients and $T$ is the sampling-time interval. The highest value of $k_{x}$ determines the smallest value of wavelength and thus, the resolution. The largest wavelength occuring determines which is called the field of view FOV, defined as ${ }^{[17]}$

$$
\mathrm{FOV}=\frac{\lambda_{\max }}{2 \pi}=\frac{2 \pi}{k_{x, \min }}=\frac{2 \pi}{\gamma G_{x} T} .
$$

The smallest wavelength in the raster, and so the resolution, is therefore

$$
\lambda_{\min }=\frac{4 \pi}{N_{r}}\left(\frac{2 \pi}{\gamma G_{x} T}\right)=\frac{4 \pi}{N_{r}} \mathrm{FOV} .
$$

Based on the formalism presented above, Figs 1 and 2 illustrate, repectively, the timing diagram and the $\mathbf{k}$ space trajectories for one of the several ways of producing a two-dimensional magnetic resonance image, the so-called gradient echo imaging sequence. After the equilibrium magnetization of the nuclear spins, contained in a given slice of the sample, is rotated to the $x y$-plane, with the use of a slice-selective RF pulse, the phase enconding and read gradients are applied for a short period of time, moving the magnetization to the point $\mathrm{A}$ in the $\mathbf{k}$-space. At this moment, the phaseenconding pulse is switched off and the read gradient is inverted, causing the magnetization to evolve in $\mathbf{k}$-space 
in the read direction to the point $B$. While the signal is being sampled, $N_{r}$ data points are colected along a single line, with constant $k_{y}$. This is named "profile". A full echo sampling corresponds to shifting the beginning of sampling to $k_{x}=-1 / 4 \pi \gamma G_{x} N_{r} T$, acquiring $N_{r}$ read points up to $k_{x}=1 / 4 \pi \gamma G_{x}\left(N_{r}-1\right) T$, the size of the profile. After $N_{p}$ repetitions of the process, with different phase encoding gradients, the whole $\mathbf{k}$-space is scanned and an array of $N_{r} \times N_{p}$ data points are acquired. $T E$ is the time of echo formation and $T R$ is the time repetition of the sequence. In CMRI, the time repetition $T R$ is of the order of the relaxation time $T_{1}$ and a typical array is a square matrix with $256 \times 256$ data points.

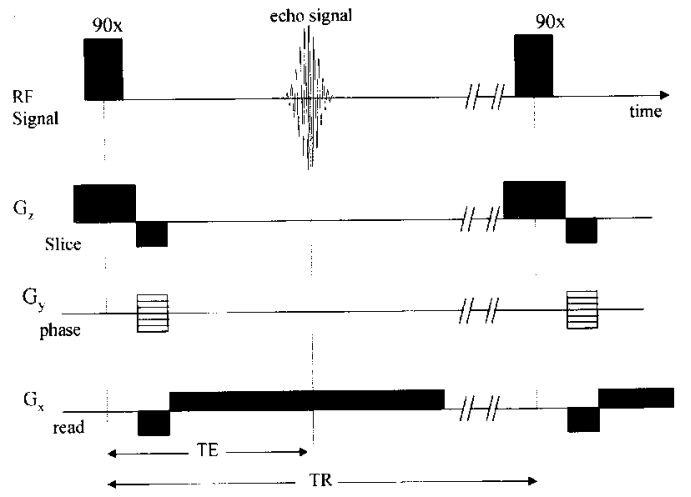

Figure.1 Gradient-echo imaging sequence. After the sliceselective excitation $90^{\circ}$ pulse, the transverse magnetization is phase-encoded with a gradient $G_{y}$ and an echo is formed in the presence of a constant reading gradient $G_{x}$. TE is the time for echo formation and TR is the time repetition of the sequence.

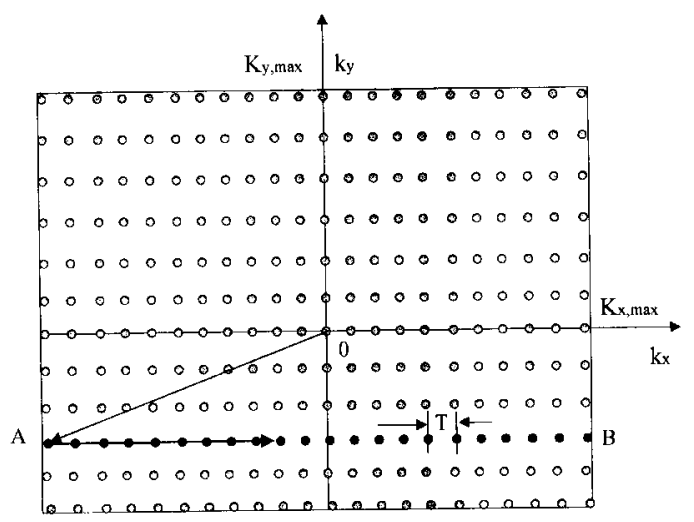

Figure.2 $K$-space trajectories of spin-echo. $T$ is the sampling time. $G_{x}$ and $G_{y}$ are gradients responsible for frequency and phase encoding, respectively. The first is termed reading gradient since the signal is acquired in its presence.

\section{Echo Planar Imaging -EPI sequences}

The echo-planar imaging technique (EPI) and related variants of the method have the hability of scanning entirely the spatial frequency $\mathbf{k}$-space, using for that the transverse magnetization produced by a single excitation of the nuclear spins in the selected slice ${ }^{[8]}$. These techniques are referred to as ultra-fast imaging sequences. The main benefits of these methods include short examination times and the visualization of noncyclic body motions. The specialized literature mention a variety of sequences of EPI, among which we will describe four of them, aiming a better understanding of these sequences since our proposal is to present a slightly modified version of the method. These sequences are: i) The FLEET (Fast Low-angle Excitation Echo planar Technique), that involves a scanning through the $\mathbf{k}$-space, in form of zig-zag ${ }^{[8,18]}$; ii) The BEST (Blipped Echo planar Single-pulse Technique), which is a sequence that scans the $\mathbf{k}$-space in a rectangular way; iii) The sequence MBEST (Modulus BEST), which is a modified version of BEST is more rapid than FLEET; iv) The last one is the spin-echo MBEST, which introduces a refocusing $180^{\circ} \mathrm{RF}$ pulse in MBEST, making it free from $\mathrm{T}_{2}^{*}$, which is the characteristic relaxation time associated to the inhomogeneity of the static magnetic field and other disturbances that increase the transverse relaxation rate $\mathrm{e}^{[8,19]}$.

As the correct raster in the $\mathbf{k}$-space consists of parallel lines, the zig-zag sweep required by FLEET needs appropriate compensation. This is accomplished by performing a double zig-zag scanning in which the second acquisition is carried out with the raster moving into the opposite quadrant, by reversing the sign of the starting $G_{y}$ gradient. The second scanning is performed immediately after the first one, without waiting for the restoration of the magnetization along the $z$-axis, as in conventional MRI. In order to ensure that the signal amplitudes are the same in both excitations, the angle of rotation due to the first $\mathrm{RF}$ pulse must be equal to $45_{x}^{0}$ while the second pulse must be equal to $90_{x}^{0}{ }^{[18]}$.

The second EPI acquisition scheme, the BEST, is more rapid than FLEET because the double sweep of $\mathbf{k}$-space is avoided. This becomes possible by ensuring that the raster in $\mathbf{k}$-space is rectangular and not in zigzag form. This is achieved by avoiding the steady $k_{x}$ evolution associated with a constant $G_{x}$ gradient. In this sequence, $G_{x}$ consists of a series of short pulses, applied soon after each $k_{y}$ blip which are responsible for the phase codification. The total image acquisition time for BEST is typically $32 \mathrm{~ms}$, which is about one half the time required for FLEET. However, the BEST sequence samples only two quadrants of $\mathbf{k}$-space, $\left(k_{x \min } \leq \mathrm{k}_{x} \leq k_{x \max }\right.$ and $\left.0 \leq \mathrm{k}_{y} \leq k_{y \text { max }}\right)$, what confers a factor of $1 / 2$ penalty in its spectrum amplitude ${ }^{[9]}$.

The timing diagram of the third echo-planar acqui- 
sition scheme, known as MBEST, is shown in Fig. 3. In this sequence the sweep of $\mathbf{k}$-space is also rectangular, as for BEST, but it samples four quadrants of $\mathbf{k}$-space, as illustrated in Fig. 4. This is achieved through the application of a set of $N_{p}$ short alternate reading gradient pulses $G_{x}$, applied soon after each phase encoder pulse gradient $G_{y}$. Also in this sequence, the transverse magnetization is produced by a single slice-selective excitation $90_{x}$ pulse. This magnetization is used to scan the k-space entirely, as long as the scanning is fast compared to the relaxation time $T_{2}$. The transverse magnetization, created by the selective pulse, is positioned in the left-lower corner of the $\mathbf{k}$-space, through a simultaneous application of a phase enconding and a read gradient for a short period of time. The phase gradient is then cut-off while the read gradient is reversed in order to form the first echo. One of the aims of the phase encoder pulses is to perform the change of lines when the $\mathbf{k}$-space is being scanned. The $N_{p}$ signals of the echoes train are created by application of successives short phase enconding pulses (blips) and alternate read gradients, so that all lines in $\mathbf{k}$-space are acquired within an acquisition window $A W$. Because in this sequence, consecutive lines in $\mathbf{k}$-space are sampled in opposite directions, the alternate data lines in the array must be reversed prior to Fourier transformation. Just as that for BEST, also for MBEST, the echoes are formed with application of the read gradients. Like the other two former sequences, this one is also subjected to $\mathrm{T}_{2}^{*}$ attenuation. However, with use of MBEST, the quality of the image is almost free from artifacts or blurring, anomalies that affect the images excellence somehow ${ }^{[18]}$. Besides, in this sequence, the data acquisition time is increased to about $64 \mathrm{~ms}$, since now the full $\mathbf{k}$-space is sampled ${ }^{[21]}$.

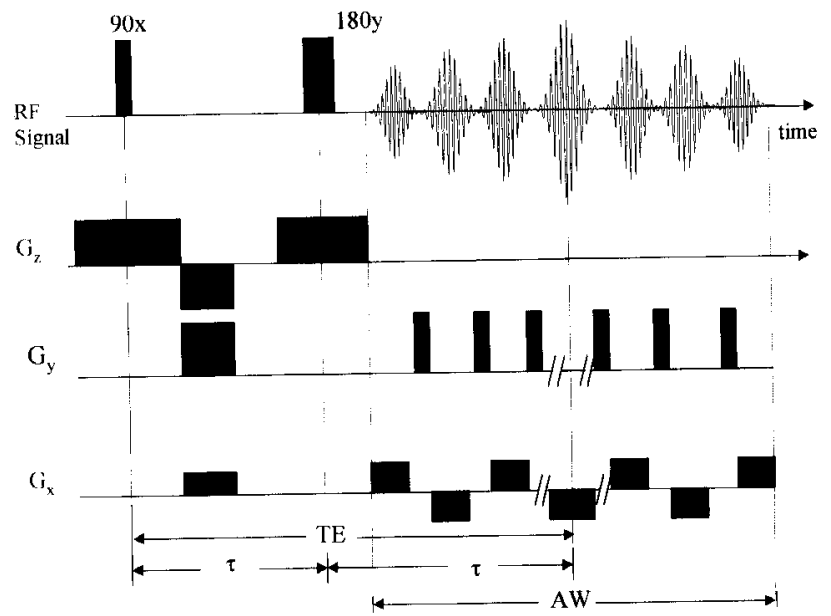

Figure.3 Echo-Planar Imaging (EPI) Sequence. The magnetization is rotated to the slice-plane by the slice-selective $90^{\circ} \mathrm{RF}$ pulse and is recalled $\mathrm{Np}$ times by alternating read gradients $G_{x}$. The whole grid in the $k$-space is scanned with a single excitation. Each individual echo is phase encoded by blipping $G_{y}$ prior to the collection data of each echo.

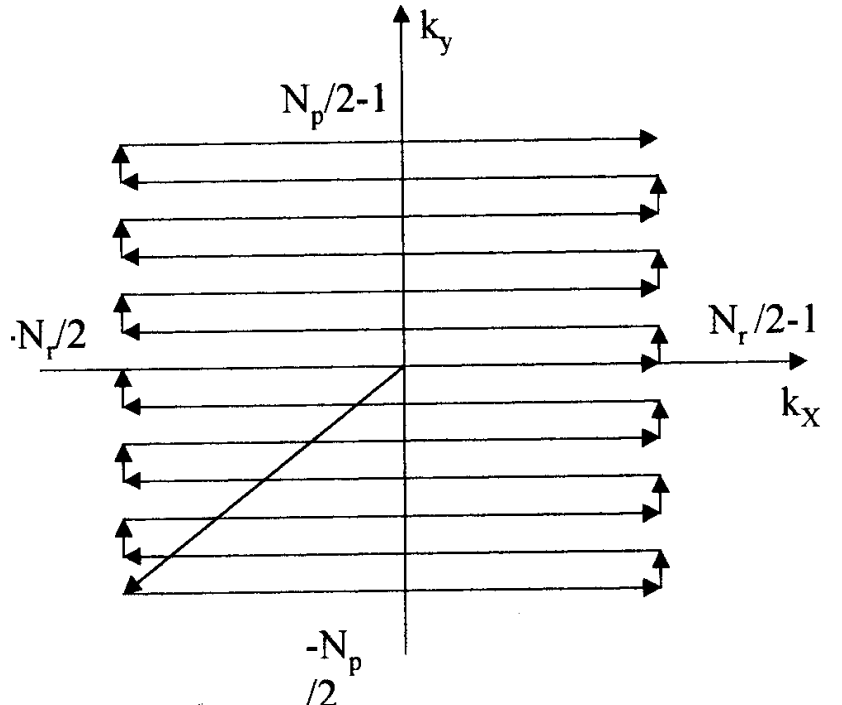

Figure.4 Echo-Planar Imaging (EPI) $k$-space raster. The magnetization is placed in the left-lower corner of the grid, using the preparation gradients $G_{x}$ and $G_{y}$.

In general terms, the main problems associated with EPI and variant methods, when compared to the conventional MRI technique, are low spatial resolution, high susceptibility to produce artifacts and poor signal/noise ratio (SNR). These effects are more accentuated in those versions which use low flip angles to rotate only a fraction the equilibrium magnetization to the transverse plane ${ }^{[11]}$.

A method to decrease or even to eliminate the effect of $T_{2}^{*}$ attenuation was proposed by Rzedzian and Pykett $[12,13]$. The method consists in the application of a $180_{y}^{0}$ $\mathrm{RF}$ refocusing pulse in the EPI sequence, applied at a time $\tau$ after the slice selective pulse. This pulse generates an effecitive echo signal at a time $2 \tau$ after the application of $90_{x}^{0}$ pulse, refocusing the spins that lost their phase coherence in the transverse magnetization due to homogeneous and inhomogeneous broadening, so that now, the amplitude of the spin-echoes are subjected only to $T_{2}$ relaxation. This sequence is illustrated in Fig. 5, while a representation for the $\mathbf{k}$-space raster is shown in Fig. 6.

In this sequence, the timing is adjusted in such a way that the center of the echo train TE (which is also the center of $\mathbf{k}$-space and the acquisition window) is positioned at time $2 \tau$ after the $90_{x}^{0} \mathrm{RF}$ pulse. As previously described, the total time of scanning the $\mathbf{k}$ - space in the EPI sequences, depends on the time for the effective echo formation $T E$ and also on the time length of the acquisition window $A W$. Complete images with an array with $128 \times 128$ data points have been acquired in times that last between 25 and $100 \mathrm{~ms}^{[12]}$. This very short imaging time makes functional studies possible and of course almost eliminates the effects of impredictable patient movements on spatial resolution ${ }^{[22]}$. 


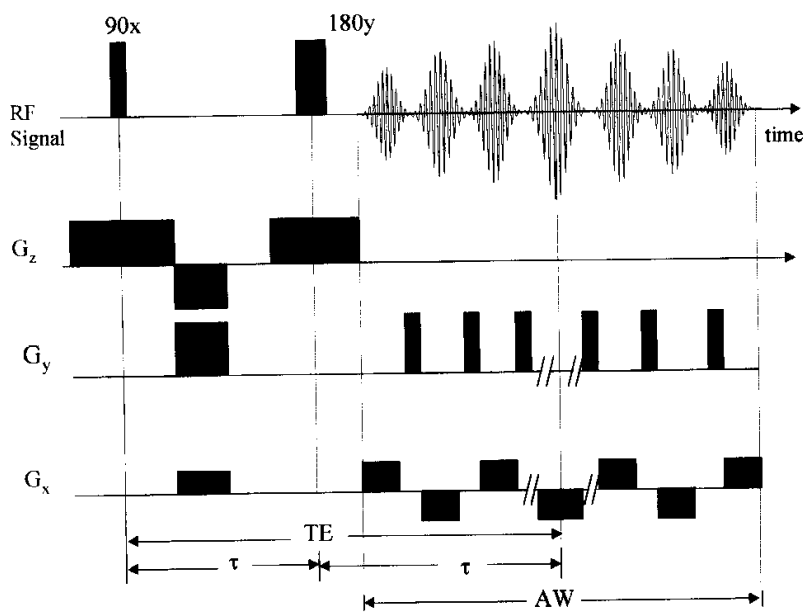

Figure.5 Echo-Planar Imaging (EPI) sequence. An $180^{\circ} \mathrm{RF}$ pulse is applied at time after the $90^{\circ} \mathrm{RF}$ excitation pulse to refocus the inhomogeneous broadening of the magnetization.

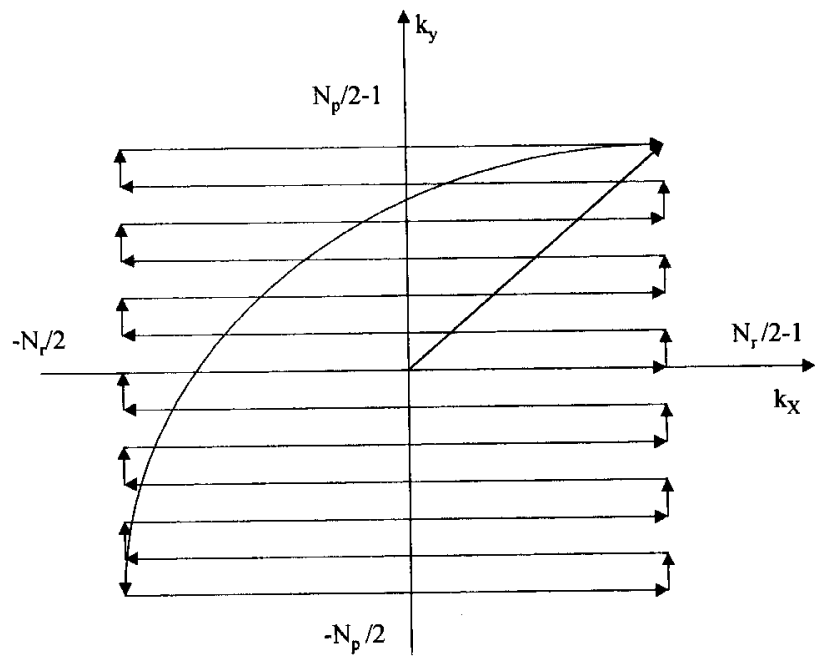

Figure.6 Echo-Planar Imaging (EPI) raster. The magnetization is place in the left-lower corner of the grid, using the preparation gradients $G_{x}, G_{y}$ and the refocusing $180^{\circ} \mathrm{RF}$ pulse.

In comparison with the conventional MRI techniques, the EPI method, unfortunately introduces at least two main technical drawbacks ${ }^{[7]}$. One is that the alternating gradient applied as series of rectangular pulses are often difficult to be implemented when the required gradient power and frequency are high ${ }^{[14]}$. Secondly, the implementation of this technique in standard commercial equipments, request the use of gradient coils capable to generate field gradients of the order of $1 \mathrm{G} / \mathrm{cm}$, with slew rates faster than $200 \mu \mathrm{s} / \mathrm{G} / \mathrm{cm}$, and a fast $\mathrm{A} / \mathrm{D}$ converter capable of handling bandwidths in excess of $300 \mathrm{kHZ}{ }^{[8]}$. Besides, the fast switching of the gradients $G_{x}$ and $G_{y}$, using high currents, induces eddy currents in the metallic structures of the equipment which, by its turn, generates artifacts, harming the quality of the image. However, improvements in gradient driving circuitry and the use of larger magnetic fields, have resulted in an increase in image array size from $32 \times 32$, in the infancy of the technique, to size of $128 \times 128$, at present days, producing acceptable image quality ${ }^{[8]}$. In particular, the effect caused in the image quality due to the appearance of eddy currents can be substantially reduced with improvements in gradient coils design ${ }^{[18]}$.

\section{Modified version of EPI se- quence}

As it was observed in the previous section, the spin echo EPI sequence is marked by the application of quite short pulses $G_{y}$ for phase codification. Another feature of BEST and MBEST sequences is the rapid gradients switching. The raising and falling times of these gradients are of the order of $100 \mu \mathrm{s}$, which confer to them a profile similar to trapezes and not a perfect square form as they are shown in Figs. 3 and 5. This effect can be compensated for by using non linear signal sampling along the $\mathrm{k}_{y}$ axis ${ }^{[9]}$.

As mentioned before, one of the main incovenients in the implementation of the EPI method in commercial systems is the demand for producing gradients of very high magnetic fields ( 1 to $40 \mathrm{G} / \mathrm{cm}$ ) and very short switching times $\left(2 \times 10^{-3}\right.$ to $\left.5 \times 10^{-4} s\right)$. Besides, there is also the problem of the eddy currents in the metallic structure of the spectrometer. Michael Stehling and co-workers $^{[23]}$, show that when very fast raising and falling times are requested, gradient coils of low inductance $(\simeq 100 \mu H)$ must be used. This demands a lot from the system of the gradient circuits, which have to supply currents up to $1000 A$ at $320 V^{[23]}$.

In this section, in an attempt of minimizing these problems, we are proposing a slightly modified version for the spin-echo MBEST sequence. As illustrated in Fig. 7, in this new version, the gradient of the phase encoding pulse $G_{y}$ is small in magnitude and maintained constant during the whole readout time. A constant time delay is placed between two consecutive alternating read gradients, in order to perform the change of lines in the $\mathbf{k}$-space array. The sampling of the $\mathbf{k}$-space is shown in Fig. 8. Since now the phase gradient pulse is constant, the $\mathbf{k}$-space grid is produced by slightly inclined lines, in form of zig-zag. In all other aspects the version is similar to the spin-echo EPI sequence. In what follows, the implication of the constant phase encoding gradient on the image reconstruction procedure will be analysed. 


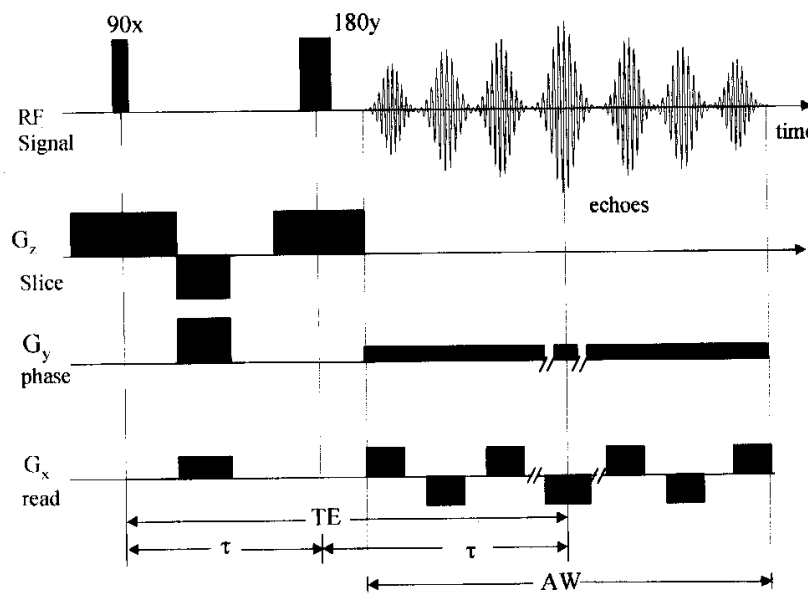

Figure.7 Modified version of the Echo-Planar Imaging (EPI) sequence. The phase encoding is performed using a constant gradient $G_{y}$ of small magnitude. An $180^{\circ} \mathrm{RF}$ pulse is applied at time $\tau$ after the slice-selective $90^{\circ}$ RF pulse to refocus the inhomageneous broadening of the magnetization.

As it is shown in figures 7 and 8 , the application of a constant phase-enconding gradient $G_{y}$ simultaneously with the read gradient $G_{x}$ leads to a non-orthogonal sampling in $\mathbf{k}$-space and therefore to modifications in the reconstruction of the image by two-dimensional Fourier transform. Due to this kind of shear effect on the grid, the trajectories in $\mathbf{k}$-space will form an small angle $\phi=\arctan \left(G_{y} / G_{x}\right)$ with respect to $x-$ axis. According to eqn.(8), $\rho(x, y)$ represents the planar spin density of the object and $S\left(k_{x}, k_{y}\right)$, the value of the NMR signal that would be measured at a given position in $\mathbf{k}$-space. For a non-rectangular grid, like the one shown in figure 8, the eqn.(8) may be modified and written as

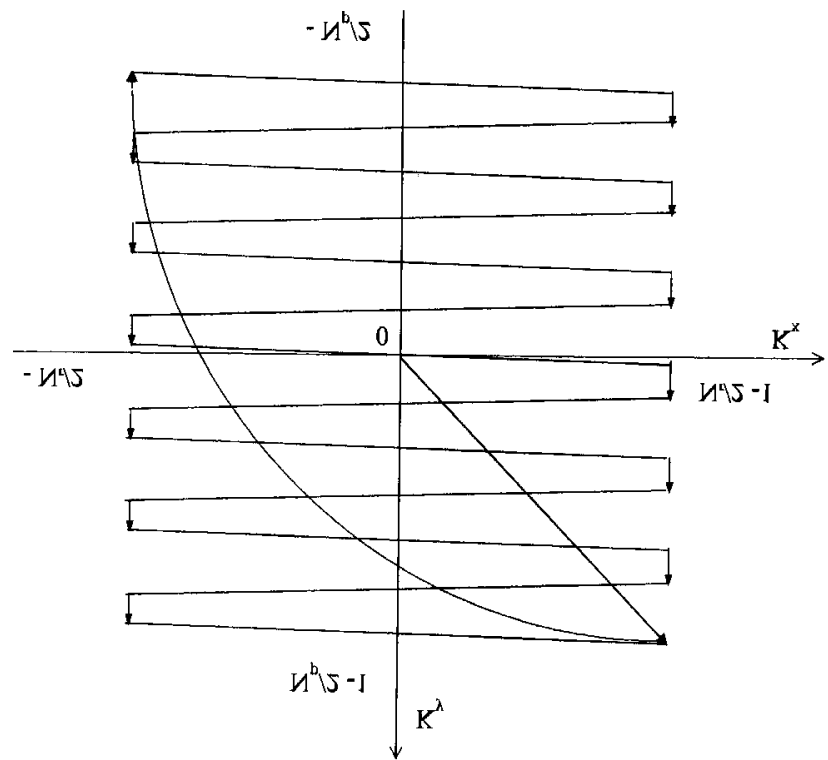

Figure.8 Modified version of the Echo-Planar Imaging (EPI) $k$-space raster. The inclined lines is due to the use of a constant phase encoding gradient $G_{y}$.

$$
\begin{aligned}
\rho^{\prime}\left(x, y, z_{0}\right)= & \iint S\left(k_{x}^{\prime}(t), k_{y}^{\prime}(t)\right) e^{-i 2 \pi\left[x k_{x}^{\prime}(t)+y k_{y}^{\prime}(t)\right]} d k_{x}^{\prime} d k_{y}^{\prime} \\
= & \iint S\left[k_{x}(t)\left(1+\frac{G_{y}^{2}}{G_{x}^{2}}\right)^{\frac{1}{2}}, k_{y}(t)\right] \\
& \times e^{-2 \pi i\left[x k_{x}(t)\left(1+\frac{G_{y}^{2}}{G_{x}^{2}}\right)^{\frac{1}{2}}+y k_{y}(t)\right]} d k_{x}^{\prime} d k_{y}^{\prime},
\end{aligned}
$$

which corresponds to the spin density distorted by the shear in the readout direction. As the NMR signal is sampled at a finite sampling rate, so that $\mathrm{N}_{x}$ points is obtained per profile, we need to change eqn. (17) to a digital form. In order to achieve this, let us define the planar-rectangular spin density $\rho(x, y)$ by ${ }^{[24]}$

$$
\rho_{l, m}=\rho(l \Delta x, m \Delta y),
$$

where $l, m$ are pixel indices and $\Delta x, \Delta y$ are pixels dimensions. For a data set measured on a rectangular grid spaced by $\Delta k_{x}=1 / N_{x} \Delta x$ and $\Delta k_{y}=1 / N_{y} \Delta y$, the value of the NMR signal that would be measured at a given position in the $\mathbf{k}$-space is

$$
S_{l, m}=S\left(\frac{1}{2 \pi} \gamma G_{x} l T, \frac{1}{2 \pi} \gamma G_{y} m T\right)
$$

and for full k-space sampling, a discrete Fourier transformation may be written as

$$
\rho_{l, m}=\sum_{l=-\frac{1}{2} N}^{\frac{1}{2} N-1} \sum_{m=-\frac{1}{2} N}^{\frac{1}{2} N-1} S_{l, m} e^{-2 \pi i\left(\frac{\ln x}{N_{x}}+\frac{m n_{y}}{N_{y}}\right)}, \quad n_{x}, n_{y}=-\frac{1}{2} N, \ldots, \frac{1}{2} N-1 .
$$


However, in our modified EPI version, the data set is sampled on a non-rectangular grid, based on a vector $\mathbf{k}_{r}$, having components

$$
k_{x}^{\prime}(t)=\frac{1}{2 \pi} \gamma G_{x} l T\left(1+\frac{G_{y}^{2}}{G_{x}^{2}}\right)^{\frac{1}{2}}, \quad k_{y}^{\prime}(t)=\frac{1}{2 \pi} \gamma G_{y} m T
$$

The Fourier transform of this data set gives the effective image

$$
\begin{aligned}
\rho_{l, m}^{\prime} & =\sum_{l=-\frac{1}{2} N} \sum_{m=-\frac{1}{2} N}^{\frac{1}{2} N-1} S\left[k_{x}^{\prime}(l, T), k_{y}^{\prime}(m, T)\right] e^{\left[\frac{1 \ln x}{N_{x}}\left(1+\frac{G_{y}^{2}}{G_{x}^{2}}\right)^{\frac{1}{2}}+\frac{m n_{y}}{N_{y}}\right]} \\
n_{x}, n_{y} & =-\frac{1}{2} N, \ldots, \frac{1}{2} N-1
\end{aligned}
$$

which corresponds to the spin density distorted by a shear in the readout direction. For single shot experiments, considering $G_{y} \ll\left|G_{x}\right|$ and with constant magnitude, we can make the approximation $k_{x^{\prime}}=k_{x}(1+$ $\left.\frac{G_{y}^{2}}{G_{x}^{2}}\right)^{\frac{1}{2}} \simeq k_{x}, k_{y}^{\prime}=k_{y}$. With this, according to eqns. (20) and (22), we will have $\rho^{\prime}\left(x, y, z_{0}\right) \simeq \rho\left(x, y, z_{0}\right)$, resulting in a shear effect negligibly small in the image reconstruction procedure.

Thus, with the elimination of the succession of the $N_{p}$ fast pulses $G_{y}$ (blips), in the conventional EPI, we aim to eliminate, at least partially, the problem of circulanting currents in the metallic stucture of the magnet and consequently, reducing the artifacts originated from the fast switching of the gradient coils related to the phase encoding pulses. Because the reading gradients $G_{x}$, in this new version, has the same form as in spin-echo EPI sequence, the problem of circulanting currents is minimized but not completely eliminated. Extra improvements could be achieved with the development of screened gradient coils ${ }^{[25,26]}$.

Besides the advantages already mentioned, obtained with the partial elimination of the inconveniences created by the circulating currents, we can still mention others that are related to the safety of the resonance exams in medical clinics. As already mentioned, the technique of EPI differs of conventional MRI by the fact that it requests gradients with high magnetic fields and very fast swichting times. According to Sethling et al $^{[23]}$, these cause high variation taxes in the gradient coils, 50 to $100 \mathrm{~T} / \mathrm{s}$, particularly when images with better resolution degree are requested. Associated with these factors, in the same reference, there are reports of recent investigations of sensations in patients, as tickles and even pain, when submitted to clinical exams. This could be called collateral effects in exams with magnetic resonance in humans. Using the procedure proposed here, these effects should be diminished, because the gradient phase encoder magnitude is small and maintained constant during the whole sampling time. However, only an experimental implementation of this new version, will garantee us wether the method is viable and if the several improvements pointed out could be confirmed in practice.

\section{Conclusions}

Trying to achieve a better understanding of the main characteristics of the echo-planar imaging (EPI) technique, in the first section of this work, we presented a brief discussion of the conventional MRI procedures for image reconstruction. The second section is dedicated to the presentation of the main aspects of four variants of the EPI method: namely, the FLEET, BEST, MBEST and another one that could be called SpinEcho MBEST, trying to show their advantages and disadvantages, as compared to the conventional MRI, in the process of fast image reconstruction procedures. Finally, in the last section, we introduced a modified version of the Spin-Echo EPI sequence. In this new version, the fast switching phase encoding gradients blips were eliminated. This modification seems pertinent and easier to be implemented than other existint EPI methods.. Other two benefits that we are expecting to achieve with the use of a constant phase encoding gradient, are: i) A possible improvement in the image quality, due to the reduction of ghosting artifacts related to the circulating currents in the metalic structure of the spectrometer. ii) The reduction of the unpleasant sensations in patients in medical clinics, associated with the use fast switching time phase encoding gradients. As discussed in section 4, a possible pratical limitation of this modified version is the introduction of a shear effect in the k-space grid, which may be considered completely negligible, provided that the magnitude of the gradient $\mathrm{G}_{y}$ is small compared with the reading gradient $\mathrm{G}_{x}$. Another source of ghosting artifacts, inherent to EPI methods, is the use of two opposite polarities readout gradients for signal acquision. The modified procedure proposed here does not overcome this sort of dificulty and proposals for additional improvements of the method are necessary. 


\section{Acknowlegments}

The authors thank Dr. Alberto Tannús of the Instituto de Física de São Carlos - Universidade de São Paulo for helpful discussions and assistance and Dr. Orlando Afonso Valle do Amaral of the Instituto de Física da Universidade Federal de Goiás for reading the manuscript. One of us (S.S.R) thanks the support of the Brazilian Agency CAPES and The Universidade Federal de Goiás.

\section{References}

[1] P. Mainsfield and P.K. Grannel. J. Phys. 66, L422 (1973).

[2] P.C. Lauterbur. Nuture. 242, 190 (1973).

[3] P.C. Lauterbur. Pure Appl. Chem. 40, (1974).

[4] P. Mansfield and P.K. Grannel. Phys. Rev. B12, 3618 (1975).

[5] I.D. Weisman et. al. Science 178, 1288 (1990).

[6] P. Mansfield, A.A. Maudsley and T. Baines. J. Phys. E: Scient. Instrum. 9, 271 (1976).

[7] C.B. Ahn, J.H. Kim and Z.H. Cho, Trans. Med. Imaging, MI-5 (1), 2 (1986).

[8] A.C. Silva and I. J. Lowe, Braz. J. Phys. 25, 404 (1995).

[9] P.T. Callaghan. The Principles of Nuclear Magnetic Resonance Microscopy, Oxford University Press, Oxford, 1991.

[10] P. Mansfield, A.A. Maudsley and T. Baines, J. Phys. E: Scient. Instrum. 9, 271 (1976).
[11] R. J. Ordidge et. al. Magn. Reson. Med. 10, 227 (1989).

[12] A.M. Howseman, et al. British Journal of Radiology, 61, $822(1988)$.

[13] A. Kumar and D. Welti and R.R. Ernst, Nuturwiss, 62, 34 (1975).

[14] A. Kumar, D. Welti and R.R. Ernst, J. Magn. Reson. 18, 69 (1975)

[15] P. Mansfield. Contemp. Phys. 17, 553 (1976).

[16] P. Mansfield, J. Phys. E. 21, 18 (1988).

[17] M.T. Vlaardingerbroek and J. A. den Boer, A textbook on Theory and Practice, Philips Medical Systems, Netherlands (1995).

[18] B. Chapman et al.. Magn. Reson. Med., 5, 246 (1987).

[19] R.R. Rzedzian and I.L. Pykett, Radiology, 161, 338 (1986).

[20] I. L. Pykett and R.R. Rzedzian, Magn. Reson. Med. 5, 553 (1987).

[21] B. Chapman et al., Magn. Reson. Med. 10, 227 (1989).

[22] C.N. Guy, Contemp. Phys. 37 (1), 15 (1996).

[23] M.K. Stehling, et. al. Radiology 171, 41 (1989).

[24] F. Hennel et.al. Magn. Reson. Med. 34, 520 (1995).

[25] J.M.S. Hutchison, R.J. Sutherland and J.R. Mallard, J. Phys. E 11, 217 (1978).

[26] P. Mansfield and B. Chapman, J. Magn. Reson. 72, 211 (1987). 